\title{
WEAK RADICAL CLASSES
}

\author{
HUDA MOHAMMED J. AL-THANI
}

\begin{abstract}
We introduce the concept of weak radical classes and proceed to construct these classes. In section 2 we define strongly austere semimodules. Theorem 1 characterizes strongly autere semimodules with respect to modular maximal subtractive ideals. We present an example of a weak radical class that can be neither a semiradical class nor a radical class. In section 4 we show that the semiradical class over a certain restricted class of semirings is equivalent to the weak radical class. We also demonstrate the construction of a semiradical class over this class. Finally, we show in example 3 that a semiradical class need not to be a radical class.
\end{abstract}

\section{Introduction}

Recall (cf. [1], [3] \& [4]) the following definitions and facts:

1.1. A non-empty subset $N$ of a left semimodule $M$ is subtractive if and only if for all $m, m^{\prime} \in M, m+m^{\prime} \in N$ and $m \in N$ imply that $m^{\prime} \in N$.

1.2. An austere semimodule $M$ is a semimodule with only two subtractive semimodules, 0 and $M$ itself.

1.3. Let $\alpha: M \longrightarrow N$ be a homomorphism of semimodules. The subsemimodule $\operatorname{Im}(\alpha)$ of $N$ is defined as

$$
\operatorname{Im}(\alpha)=\left\{n \in N: n+\alpha\left(m^{\prime}\right)=\alpha(m) \text { for some } m, m^{\prime} \in M\right\} .
$$

Then $\alpha$ is $i$-regular if $\alpha(m)=\operatorname{Im}(\alpha) ; \alpha$ is $k$-regular if for any $m, m^{\prime} \in M, \alpha(m)=\alpha\left(m^{\prime}\right)$ implies $m+k=m^{\prime}+k^{\prime}$ for some $k, k^{\prime} \in \operatorname{Ker} \alpha$; and $\alpha$ is regular if it is both $i$-regular and $k$-regular.

1.4. Let $\mu$ be a class of semimodules and $M$ be a semimodule. The reject of $\mu$ in $M$ and the strong reject of $\mu$ in $M$ are defined as $\operatorname{Re} j_{M}(\mu)=\cap\{\operatorname{Ker} h \mid h: M \longrightarrow U$ for some $U \in \mu$ and $h$ is a homomorphism $\} ;$ sts $\operatorname{Re} j_{M}(\mu)=\cap\{\operatorname{Ker} h \mid h: M \longrightarrow U$ for some $U \in \mu$ and $h$ is a regular homomorphism\}, respectively.

Received June 23, 2003; revised March 30, 2004.

2000 Mathematics Subject Classification. 16Y60.

Key words and phrases. Weak radical class, semiradical class, strongly austere semimodules, modular ideals.

The author records appreciation to Professor Herbert C. for comments and advice that improved this presentation. Dedicated to the capture AL Aqsa mosque. 
1.5. Let $M$ be a left $R$-semimodule. An element $m \in M$ is regular if the homomorhphism $\theta: R \longrightarrow M$ defined by $r \mapsto r m$ is a regular homomorphism. Note that $M$ is regular if for every $m \in M, m$ is regular.

1.6. If $M$ is a left $R$-semimodule, then its left annihilator is $L_{R}(M)=\{r \in R: r m=0$ for every $m \in M\}$ and its left strong annihilator is $S L(M)=\{r \in R: r m=0$ for every regular element $m \in M\}$.

1.7. A semiring $R$ is said to be $k$-homomorphic image of $S$ if there exists a $k$-regular homomorphism from $S$ onto $R$.

1.8. An ideal $I$ of a semiring $R$ determines an equivalence relation $\equiv_{I}$ on $I$, the Bourne relation, defined as $r \equiv_{I} r^{\prime}$ if and only if there exist elements $a, a^{\prime} \in I$ satisfying $r+a=r^{\prime}+a^{\prime}$. If $r \in R$ then we write $r / I$ instead of $r / \equiv_{I}$. The factor semiring $R / \equiv_{I}$ is denoted by $R / I$.

1.9. A left ideal $I$ in a semiring $R$ is regular if the left $R$-semimodule $R / I$ is regular. A semiring $R$ is regular if $\{0\}$ is regular.

1.10. Let $\rho$ be a class of semirings. A semiring (ideal) belonging to the class $\rho$, will be called a $\rho$-semiring ( $\rho$-ideal).

1.11. A class $\rho$ of semiring is a radical class whenever the following three conditions are satisfied:

(a) $\rho$ is homomorphically closed; i.e. if $S$ is a homomorphic image of a $\rho$-semiring $R$, then $S$ is also a $\rho$-semiring

(b) Every semiring $R$ contains a $\rho$-ideal $\rho(R)$ which in turn contains every other $\rho$-ideal of $R$

(c) The factor semiring $R / \rho(R)$ does not contain any nonzero $\rho$-ideal; i.e. $\rho(R / \rho(R))=0$.

1.12. A class $\bar{\rho}$ of semirings is said to be a semiradical class whenever the following three conditions are satisfied:

$\left(\mathrm{a}^{\prime}\right) \bar{\rho}$ is $k$-homomorphically closed; i.e. if $S$ is an $k$-homomorphic image of a $\bar{\rho}$-semiring $R$, then $S$ is also a $\bar{\rho}$-semiring

$\left(\mathrm{b}^{\prime}\right)$ Every semiring $R$ contains a $\bar{\rho}$-subtractive ideal $\bar{\rho}(R)$ which in turn contains every other $\bar{\rho}$-subtractive ideal of $R$

$\left(\mathrm{c}^{\prime}\right)$ The factor semiring $R / \bar{\rho}(R)$ does not contain any non-zero $\bar{\rho}$-subtractive ideals; i.e. $\bar{\rho}(R / \bar{\rho}(R))=0$.

For example, the class $\sigma$ of all left quasi-regular semirings forms both a radical class and a semiradical class, where $R$ is left quasi-regular if for every element $x \in R$ there exists an $r \in R$ such that $r+r x+x=0$.

A semiring that does not contain any non-zero $\bar{\rho}$-subtractive ideals is termed $\bar{\rho}$ semisimple.

Finally we mention the following two propositions. 
Proposition 1. If I is a subtractive ideal of $R$, then there is a one-to-one correspondence between subtractive ideals $H$ of $R$ that contain $I$ and subtractive ideals $J$ of $R / I$. Therefore every subtractive ideal in $R / I$ is of the form $H / I$, where $H$ is a subtractive ideal of $R$ that contains $I$.

Proof. Let $I$ be a subtractive ideal of $R$ and let $J$ be a subtractive ideal of $R / I$. Let $H=\{x \in R: x / I \in J\}$. If $x, y \in H$ and $r \in R$ then $x / I+y / I=x+y / I \in J$ and $r / I . x / I=r x / I \in J$. Therefore $H$ is an ideal of $R$ that contains $I$. If $x, x+r \in H$ then $x / I, x+r / I \in J$. Since $J$ is subtractive, $r / I \in J$, so $r \in H$. Thus $H$ is a subtractive ideal. Let $S(R)$ be the set of all subtractive ideals of $R$ containing $I$ and let $S(R / I)$ be the set of all subtractive ideals of $R / I$. Define $\theta: S(R / I) \rightarrow S(R)$ by $\theta(J)=H$. Let $\theta(J)=\theta\left(J^{\prime}\right)$. If $x / I \in J$ then $x \in H$, so $x / J \in J^{\prime}$. Therefore $J=J^{\prime}$. If $K$ is a subtractive ideal of $R$ containing $I$, then $\theta(K / I)=K$. Thus there is one-to-one correspondence between the subtractive ideals $H$ of $R$ that contain $I$ and the subtractive ideals $J$ of $R / I$.

Proposition 2. If $\bar{\rho}$ is a semiradical, then $R$ is an $\bar{\rho}$-semiradical semiring if and only if $R$ cannot be mapped $k$-regular homomorphically onto a non-zero $\bar{\rho}$-semisimple semiring.

Proof. If $R$ is an $\bar{\rho}$-semiradical semiring then condition $\left(\mathrm{a}^{\prime}\right)$ implies that every non zero $k$-regular homomorphic image of $R$ is also a $\bar{\rho}$-semiradical semiring and therefore it cannot be $\bar{\rho}$-semisimple. Conversely, if $R$ is not a $\bar{\rho}$-semiradical semiring, then by $\left(\mathrm{b}^{\prime}\right)$ and $\left(\mathrm{c}^{\prime}\right)$ it can be mapped $k$-regular homomorphically noto the non-zero $\bar{\rho}$-semisimple semiring $R / \bar{\rho}(R)$, where $\bar{\rho}(R)$ is the $\bar{\rho}$-semiradical of $R$.

Remark 1. For potential applications, we note that semimodules over semirings are important in studying the properties of semirings, and the latter arise in such diverse eareas of applied mathematics as optimization theory, automata theory, mathematical modeling and parallel computation systems (c.f. [4], pp iv, 138).

\section{Weak Radical Classes}

Definition 1. A class $\sigma$ of semirings is a weak semiradical class whenever the following two conditions are satisfied:

(A) If $R$ is an $\sigma$-semiring, then every non-zero $k$-regular homomorphic image of $R$ has a non-zero $\sigma$-subtractive ideal;

(D) If every-non-zero $k$-regular homomorphic image of a semiring $R$ has a non-zero $\sigma$ subtractive ideal then $R$ is an $\sigma$-semiring.

Remark 2. Observe that by axiom (D) the semiring 0 is always a $\sigma$-weak radical semiring. Furthermore, there are two trivial weak radical classes, the semiring 0 and the class of all semirings. Note that when $\sigma$ is a class of rings, this definition defaults to the standard definition of a radical class. 
The next result shows that weak semiradical is a generalization of semiradical.

Proposition 3. Every semiradical calss is weak semiradical.

Proof. If $\rho$ is a semiradical class, then the class $\rho$ satisfies conditions $\left(\mathrm{a}^{\prime}\right),\left(\mathrm{b}^{\prime}\right)$, and $\left(\mathrm{c}^{\prime}\right)$. Condition $\left(\mathrm{a}^{\prime}\right)$ implies condition $(\mathrm{A})$ trivialy. If $R$ is not a $\rho$-semiring, then by $\left(\mathrm{b}^{\prime}\right)$ we have $\rho(R) \neq R$, and so $R / \rho(R)$ is a non-zero homomorphic image of $R$ which does not contain non-zero $\rho$-subtractive ideals by $\left(\mathrm{c}^{\prime}\right)$. Thus condition (D) is also fulfilled.

Now we present some new definitions and results that will enable us to describe an example of a weak radical class.

Definition 2. A left $R$-semimodule $M$ is strongly austere if $M$ is austere and $S L_{R}(M) \neq R$.

Definition 3. A left ideal $I$ in a semiring $R$ is modular if there exists an $e \in R$ such that $r / I=r e / I$ for every $r \in R$.

Remark 3. Note that these default to the customary definitions if $M$ is a module or if $R$ is a ring.

The following theorem is analogous to the one that characterizes simple modules with maximal modular ideals.

Theorem 1. A left $R$-semimodule $M$ over a semiring $R$ is strongly austere if and only if $M$ is isomorphic to $R / I$ for some modular maximal subtractive left ideal $I$.

Proof. Let $M$ be a strongly austere semimodule. Hence $S L_{R}(M) \neq R$, so there exists a regular element $0 \neq m \in M$. Since $R m$ is subtractive, $M=R m$. Define $\theta: R \rightarrow R m$ as $\theta(r)=r m$. Since $m$ is regular, $\theta$ is a $k$-regular surjective homomorphism. Consider $\phi: R / \operatorname{Ker} \theta \rightarrow R m$ as defined by $\phi(r / \operatorname{Ker} \theta)=r m$. Clearly $\phi$ is an isomorphism.

Since $M$ is austere, $\operatorname{Ker} \theta$ is a maximal subtractive left ideal. Now $M=R m$, where $m=e m$ for some $e \in R$. Thus, for any $r \in R, r m=r e m$. By the regularity of $m$, $r+k=r e+k^{\prime}$ and $k m=k^{\prime} m=0$, hence $r e / \operatorname{Ker} \theta=r / \operatorname{Ker} \theta$. Thus $\operatorname{Ker} \theta$ is a modular maximal subtractive left ideal. Conversely, let $I$ be a modular maximal subtractive ideal of $R$ such that $M \simeq R / I$. Clearly $M$ is austere, so it suffices to prove that $S L_{R}(M) \neq R$. Suppose that $S L_{R}(M)=R$. Since $I$ is modular, there exists an element $e / I$ such that $r e / I=r / I$. Clearly $e / I$ is a regular element. Hence $R(e / I) \neq 0$ and $S L_{R}(M) \neq R$.

Corollary 1. If $R$ is a semiring, then $R$ has a strongly austere left $R$-semimodule if and only if $R$ has a modular maximal subtractive left ideal.

Proposition 4. Let $R$ and $S$ be semirings and let $\phi: R \rightarrow S$ be a surjective $k$-regular homomorphism. Then every $S$-strongly austere semimodule is an $R$-strongly austere semimodule.

Proof. Let $M$ be an $S$-strongly austere semimodule. Define $\bar{\phi}: R \times M \rightarrow M$ by the rule $r m=\phi(r) m$. Clearly $M$ is an $R$-semimodule. Let $K$ be a subtractive 
$R$-subsemimodule. Since $\phi$ is surjective, $S K=\phi(R) K=R K \subset K$. Hence $K$ is a subtractive $S$-subsemimodule. Therefore $M$ is an austere $R$-semimodule. Since $M$ is a strongly austere $S$-semimodule, there exists a regular element $0 \neq m \in M$. If $r_{1} m=r_{2} m$, then $\phi\left(r_{1}\right) m=\phi\left(r_{2}\right) m$. Since $m$ is regualr, $\phi\left(r_{1}\right)+s_{1}=\phi\left(r_{2}\right)+s_{2}$, where $s_{1} m=s_{2} m=0$ and $s_{1}, s_{2} \in S$. Since $\phi$ is onto, $s_{1}=\phi\left(r_{3}\right), s_{2}=\phi\left(r_{4}\right)$. Hence $\phi\left(r_{1}+r_{3}\right)=\phi\left(r_{2}+r_{3}\right)$. Since $\phi$ is $k$-regular, $r_{1}+r_{3}+k_{1}=r_{2}+r_{4}+k_{2}$, where $r_{1}, r_{2}, r_{3}, r_{4} \in R$ and $k_{1}, k_{2} \in \operatorname{Ker} \phi$. Clearly $\left(r_{3}+k_{1}\right) m=\phi\left(r_{3}+k_{1}\right) m=\phi\left(r_{3}\right) m+\phi\left(k_{1}\right) m=\phi\left(r_{3}\right) m+0=\phi\left(r_{3}\right) m=s_{1} m=0$. Similarly $\left(r_{4}+k_{2}\right) m=0$. If $r_{1} m+b=r_{2} m$ then $\phi\left(r_{1}\right) m+b=\phi\left(r_{2}\right) m$. Since $m$ is a regular element, $b=s_{2} m$. Since $\phi$ is onto, $s_{2}=\phi\left(r_{3}\right)$. Thus $b=\phi\left(r_{3}\right) m=r_{3} m$ and therefore $M$ is an $R$-strongly austere semimodule.

Definition 4. The Jacobson weak radical $\hat{\sigma}$ is defined as $\hat{\sigma}=\{R: R$ is commutative and has no modular maximal subtractive left ideals $\}$.

Theorem 2. The class $\hat{\sigma}$ is a weak radical class.

Proof. Let $R$ be a $\hat{\sigma}$-semiring and $S \neq 0$ a $k$-homomorphic image of $R$. Suppose that $S \notin \hat{\sigma}$. Then there exists a strongly austere left $S$-semimodule, say $M$. By proposition 4 , $M$ is an $R$-strongly austere $R$-semimodule and $M \neq 0$. By theorem $1, M$ is isomorphic to $R / I$ for some modular maximal subtractive left ideal $I$ of $R$. This contradicts the assumption that $R \in \hat{\sigma}$. Thus $S \in \hat{\sigma}$, proving the validity of (A). Next, assume that every non-zero homomorphic image of a semiring $R$ has a non-zero $\hat{\sigma}$-subtractive ideal. If $R \notin \hat{\sigma}$ then there exists a modular subtractive left ideal $I$ such that $R / I$ is a strongly austere left $R$-semimodule. By our assumption $R / I$ has a non-zero $\hat{\sigma}$-subtractive ideal. Since $R / I$ is austere, $R / I \in \hat{\sigma}$. Therefore $R / I$ has no modular maximal subtractive left ideal. But $I$ is a modular subtractive left ideal of $R / I$. Thus $R \in \hat{\sigma}$. Hence condition (D) is also satisfied and $\hat{\sigma}$ is weak radical class.

In ring theory the weak radical class coincides with the both radical class and the semiradical class. However, the next two examples will show that a weak radical class $\hat{\sigma}$ may be neither a semiradical class nor a radical class.

Example 1. Let $R=\{0, a, b, c, d, e\}$ and define operations + and $\cdot$ on $R$ as follows:

\begin{tabular}{|c|c|c|c|c|c|c|}
\hline+ & 0 & $a$ & $b$ & $c$ & $d$ & $e$ \\
\hline 0 & 0 & $a$ & $b$ & $c$ & $d$ & $e$ \\
\hline$a$ & $a$ & $a$ & $a$ & $e$ & $e$ & $e$ \\
\hline$b$ & $b$ & $b$ & $b$ & $e$ & $e$ & $e$ \\
\hline$c$ & $c$ & $e$ & $e$ & $c$ & $c$ & $e$ \\
\hline$d$ & $d$ & $e$ & $e$ & $d$ & $d$ & $e$ \\
\hline$e$ & $e$ & $e$ & $e$ & $e$ & $e$ & $e$ \\
\hline
\end{tabular}

\begin{tabular}{|l|l|l|l|l|l|l|}
\hline$\cdot$ & 0 & $a$ & $b$ & $c$ & $d$ & $e$ \\
\hline 0 & 0 & 0 & 0 & 0 & 0 & 0 \\
\hline$a$ & 0 & $b$ & $b$ & 0 & 0 & $b$ \\
\hline$b$ & 0 & $b$ & $b$ & 0 & 0 & $b$ \\
\hline$c$ & 0 & 0 & 0 & $c$ & $c$ & $c$ \\
\hline$d$ & 0 & 0 & 0 & $c$ & $c$ & $c$ \\
\hline$e$ & 0 & $b$ & $b$ & $c$ & $c$ & $e$ \\
\hline
\end{tabular}

Clearly $\{0\},\{0, a, b\},\{0, c, d\}$ and $R$ are all subractive ideals of $R$. It can be shown that $R \notin \hat{\sigma}$ and $\{0, a, b\},\{0, c, d\} \in \hat{\sigma}$. Thus $\{0, a, b\}+\{0, c, d\} \notin \hat{\sigma}$. Therefore $\hat{\sigma}$ cannot be a semiradical class. 
Example 2. Let $R$ be the semiring consisting of the three elements $0, b$ and $a$ with the two operations given by the tables

\begin{tabular}{|c|c|c|c|}
\hline+ & 0 & $b$ & $a$ \\
\hline 0 & 0 & $b$ & $a$ \\
\hline$b$ & $b$ & $b$ & $b$ \\
\hline$a$ & $a$ & $b$ & $a$ \\
\hline
\end{tabular}$\quad$\begin{tabular}{|c|c|c|c|}
\hline. & 0 & $b$ & $a$ \\
\hline 0 & 0 & 0 & 0 \\
\hline$b$ & 0 & $b$ & $b$ \\
\hline$a$ & 0 & $b$ & $b$ \\
\hline
\end{tabular}

Clearly $\{0\},\{0, b\}$ and $R$ are all left ideals of $R,\{0, b\}$ is not a subtractive left ideal and $\{0\}$ is a subtractive left ideal which is not modular. Thus $R$ has no modular subtractive left ideals. Therefore $R \in \hat{\sigma}$. Now consider the semiring $R /\{0, b\}$. Clearly 0 is a modular subtractive ideal of $R /\{0, b\}$. Hence $R /\{0, b\} \notin \hat{\sigma}$. Now $R /\{0, b\}$ is a homomorphic image of the $\hat{\sigma}$-semiring $R$, which is not a $\hat{\sigma}$-semiring. Thus the class $\hat{\sigma}$ is weak radical, not radical.

\section{Construction of a Weak Radical Class}

In thie section we develop weak radical classes in the same way that radical classes of rings are developed in [2].

Theorem 3. Let $\mu$ be any class of semiring which satisfies the following condition:

(E) Every non-zero subtractive ideal of a semiring of $\mu$ can be mapped $k$-homomorphically onto some non-zero semiring of $\mu$.

Then the class $\sigma_{\mu}=\{R: R$ cannot be mapped k-homomorphically onto any non-zero semiring of $\mu\}$ is a weak radical class.

Proof. Let $\mu$ be any class of semirings which satisfies condition (E). If $\sigma_{\mu}$ is defined as above, we shall show $\sigma_{\mu}$ is a weak radical class by showing that it meets conditions $(\mathrm{A})$ and (D). Condition (A) is clear, for whenever a $k$-homomorphic image of a semiring $R$ can be mapped $k$-homomorphcially onto a non-zero semiring of $\mu$, then $R$ itself can be mapped $k$-homomorphically onto this same semiring of $\mu$. In this case, $R$ is not a $\sigma_{\mu}$-semiring, and this establishes (A). To show that (D) holds, let $R$ be a semiring such that every non-zero $k$-homomorphic image of $R$ has a non-zero $\sigma_{\mu}$-subtractive ideal. If $R$ is itself not a $\sigma_{\mu}$-semiring, then it can be mapped homomorphically onto a non-zero semiring $R^{\prime}$ of $\mu$. By our assumption, $R^{\prime}$ must have a non-zero $\sigma_{\mu}$-subtractive ideal $I$. By (E), $I$ can be mapped homomorphically onto some non-zero semiring of $\mu$, which contradicts the definition of a $\sigma_{\mu}$-semiring. Thus $R$ must be a $\sigma_{\mu}$-semiring and (D) holds, so $\sigma_{\mu}$ is a weak radical class.

Remark 4. If we let $\mu$ be a class of strongly austere semirings, then $\mu$ satisfies (E) and we have a weak radical class as promised above. 


\section{Semiradical Classes over a Restricted Class of Semirings}

In this section we consider the class $\Im$ of semirings. It will be shown that in this class, semiradical and weak radical are equivalent. Thus by the previous section, the semiradical class can be constructed over the class $\Im$.

The following results are necessary to prove theorem 6 .

Theorem 4. (Second Isomorphism Theorem): If I and $J$ are subtractive ideals in a semiring $R$ for which $i \underset{\bar{J}}{\equiv} i^{\prime}$ implies $i \underset{I \cap J}{\equiv} i^{\prime}$, then there exists an isomorphism of semirings $I /(I \cap J) \simeq I+J / J$.

Proof. Consider the homomorphism $f: I \rightarrow I+J / J$ defined by $f(i)=i / J$. Clearly $f$ is surjective $k$-regular with kernel $I \cap J$, so $I /(I \cap J) \simeq I+J / J$.

Theorem 5. (Third Isomorphism Theorem): If $H$ is a subtractive ideal of $R$ and $K$ is an ideal of $R$ such that $K \subset H$, then $H / K$ is a subtractive ideal of $R / K$ and $(R / K) /(H / K) \simeq R / H$.

Proof. Clearly $H / K$ is subtractive ideal of $R / I$. Define $\theta: R / K \rightarrow R / H$ by $\theta(r / K)=r / H$. Now $\theta$ is a homomorphism with kernel $H / K$. If $r / H=r^{\prime} / H$, then $r+h=r^{\prime}+h^{\prime}$, so $r+h+k=r^{\prime}+h^{\prime}+k$. Hence $r / K+h / K=r^{\prime} / K+h^{\prime} / K$, so $\theta$ is $k$-regular and $(R / K) /(H / K) \simeq R / H$.

Let $\Im$ be the class of semirings satisfying the following two conditions:

$\left.{ }^{*}\right)$ If $I$ and $J$ are subtractive ideals with $i \overline{\bar{J}} i^{\prime}$ for $i, i^{\prime} \in I$, then $i \underset{I \cap}{\equiv} i^{\prime}$.

(**) If $I$ and $J$ are subtractive ideals of $R$, then $I+J$ is subtractive.

Remark 5. Note that all classes of rings are contained in the class $\Im$.

Theorem 6. A class $\sigma$ of $\Im$ is semiradical if and only if $\sigma$ is weak semiradical.

Proof. Let $\sigma$ be a semiradical class. By proposition 4, $\sigma$ is weak radical. Conversely, suppose $\sigma$ is a weak radical class of $\Im$. Let $R$ be a $\sigma$-semiring and $S \neq 0$ a $k$-regular homomorphic image of $R$. Since every $k$-regular homomorphic image of $S$ is at the same time a $k$-regular homomorphic image of $R$, by (A) every non-zero $k$-regular homomorphic image of $S$ contains a non-zero $\sigma$-subtractive ideal. Hence applying (D) we see that $S$ is a $\sigma$-semiring, establishing $\left(\mathrm{a}^{\prime}\right)$. To show $\left(\mathrm{b}^{\prime}\right)$, we have to show the sum $J=\sum_{\alpha} I_{\alpha}$ of all $\sigma$-subtractive ideals $I_{\alpha}$ of a semiring $R$ is again a $\sigma$-subtractive ideal of $R$. If $J=0$, then by (D), $J$ is a $\sigma$-subtractive ideal. If $J \neq 0$, consider an arbitrary non-zero $k$-regular homomorphic image $J^{\prime}$ of $J$. By the homomorphism theorem we may assume that $J^{\prime} \simeq J / K$, where $K \neq J$ is a subtractive ideal of $J$. By the defintion of $J$ there exists a $\sigma$-subtractive ideal $I_{\alpha}$ of $R$ such that $I_{\alpha}$ is not isomorphic to $K$. Now the second isomorphism theorem yields

$$
0 \neq\left(I_{\alpha}+K\right) / K \simeq I_{\alpha} /\left(K \cap I_{\alpha}\right)
$$


and the right-hand side, as a $k$-regular homomorphic image of the $\sigma$-semiring $I_{\alpha}$, is a $\sigma$-semiring by $\left(\mathrm{a}^{\prime}\right)$. Thus $J / K$ contains a non-zero $\sigma$-subtractive ideal $\left(I_{\alpha}+K\right) / K$ and (D) implies $J$ is a $\sigma$-subtractive ideal. Finally, we establish $\left(\mathrm{c}^{\prime}\right)$. Because we have already verified $\left(\mathrm{b}^{\prime}\right)$, we can let $J=\sigma(R)$. Now assume that $\left(\mathrm{c}^{\prime}\right)$ does not hold and let $L^{\prime}$ be a non-zero $\sigma$-subtractive ideal of the factor semiring $R / \sigma(R)$. By proposition 1 $L^{\prime}$ can be written as $L^{\prime}=L / \sigma(R)$ where $L$ is a subtractive ideal of $R$. Let $L / T$ be a $k$-regular homomorphic image of $L$. Now either $\sigma(R) \subseteq T$ or not. In the first case, the third isomorphism theorem implies $0 \neq L / T \simeq(L / \sigma(R)) /(T / \sigma(R))$, and the right-hand side, as a $k$-regular homomorphic image of the $\sigma$-semiring $L^{\prime}=L / \sigma(R)$, is a $\sigma$-semiring. Hence $L / T$. contains a non-zero $\sigma$-subtractive ideal, namely itself. In the second case, by the second isomorphism theorem, we have $0 \neq(T+\sigma(R)) / T \simeq \sigma(R) /(T \cap \sigma(R))$ and the right-hand side is again a $\sigma$-semiring because of $\left(\mathrm{a}^{\prime}\right)$ and $\sigma(R) \in \sigma$. Furthermore, $T+\sigma(R) / T$ is a subtractive ideal of $L / T$. Thus in both case the arbitrarily chosen non-zero $k$-regular homomorphic image of $L / T$ contains a non-zero $\sigma$-subtractive ideal. Applying (D) we get $L \in \sigma$, so $L$ is a $\sigma$-subtractive ideal of $R$ that is not contained in $\sigma(R)$, contradicting the definition of $\sigma(R)$. Hence condition $\left(\mathrm{c}^{\prime}\right)$ is satisfied.

In ring theory, a semiradical class is equivalent to a radical class. However, the above theorem enables us to construct the following example of a semiradical class that is not radical.

Example 3. Let $R$ be the semiring defined in example 2. Clearly $R \in \Im$. Let $\tilde{\sigma}=\{R \in \Im: R$ is commutative and $R$ has no modular maximal subtractive left ideal $I\}$. By theorem 2 and theorem $6 \tilde{\sigma}$ is semiradical class. By example 2 we see that $\tilde{\sigma}$ is semiradical class over $\Im$ that is not radical.

The following fundamental result is required to construct semiradical classes.

Theorem 7. A class $\mu \subset \Im$ is a class of all $\rho$-semisimple semirings with respect to some semiradical class $\rho$ over the class $\Im$ if and only if $\mu$ satisfies the following conditions:

(E) Every non-zero subtractive ideal of a semiring of $\mu$ can be mapped $k$-homomorphically onto some non-zero semiring of $\mu$.

(F) If every non-zero subtractive ideal of a semiring $R$ can be mapped $k$-homomorphically onto some non-zero semiring of $\mu$, then the semiring $R$ must be in $\mu$.

Proof. Suppose that we are given a semiradical class $\rho$ over the class $\Im$ and that $\mu$ is the class of all $\rho$-semisimple semirings over $\Im$. Let $R$ be in $\mu$ and let $I$ be a subtractive ideal of $R$. Since $R$ is $\rho$-semisimple, $I$ is not a $\rho$-semiradical semiring. By Proposition 2, $I$ can be mapped $k$-homomorphically onto a non-zero $\rho$-semisimple semiring; i.e., onto a semiring of $\mu$. Thus $\mu$ satisfies (E). To show that (F) is satisfied, let $R$ be a semiring that is not $\rho$-semisimple, so $R$ has a non-zero $\rho$-semiradical subtractive ideal $H$. By proposition 2 , this subtractive ideal $H$ cannot be mapped $k$-homomorphically onto a non-zero $\rho$-semisimple semiring, so our desired conclusion follows.

Conversely, let $\mu$ be any class of semirings over $\Im$ which meets conditions $(\mathrm{E})$ and $(\mathrm{F})$. By Theorem 3 the class $\rho_{\mu}=\{R \in \Im: R$ cannot be mapped $k$-homomorphically onto 
any non-zero semiring of $\mu$ \} is a weak radical class. By theorem $6 \rho_{\mu}$ is semi-radical. It remains to show that $\mu$ is the class of all $\rho_{\mu}$-semisimple semirings. If $R$ is a semiring of $\mu$, then by (E) a non-zero subtractive ideal $R$ cannot be a $\rho_{\mu}$-semiring. Thus every semiring in $\mu$ is $\rho_{\mu}$-semisimple. On the other hand, if $R \in \Im$ is any $\rho_{\mu}$-semisimple semiring then no non-zero subtractive ideal of $R$ is a $\rho_{\mu}$-semiring and therefore $R$ cannot be mapped $k$-homomorphically onto a non-zero semiring of $\mu$. By $(\mathrm{F}), R$ is itself in $\mu$. Thus $\mu$ is precisely the class of all $\rho_{\mu}$-semisimple semirings over $\Im$, and the theorem is proved.

If we can exhibit a class of semiring in the class $\Im$ that satisfy (E) and (F), we will have a semiradical class as above. If we find a class of semirings satisfying only $(\mathrm{E})$, we can use the following proposition to build it up to a class satisfying both (E) and (F).

Proposition 5. If a class $\mu \subset \Im$ of semirings satisfies (E) and if the class $\bar{\mu}$ is defined as the set of all semirings $R \subset \Im$ such that every non-zero subtractive ideal of $R$ can be mapped $k$-homomorphically onto some non-zero semiring of $\mu$, then the class $\bar{\mu}$ satisfies $(\mathrm{E})$ and $(\mathrm{F})$ as well.

Proof. Because $\mu$ satisfies (E), $\mu$ is contained in $\bar{\mu}$. If $R$ is in $\bar{\mu}$, then every non-zero subtractive ideal of $R$ can be mapped $k$-homomorphically onto some non-zero semiring of $\mu$ and thus onto a non-zero semiring $R^{\prime}$ of $\mu$. Thus $\bar{\mu}$ satisfies (E). On the other hand, let $R$ be a semiring such that every non-zero subtractive ideal of $R$ can be mapped $k$-homomorphically onto a non-zero semiring $R^{\prime}$ of $\bar{\mu}$. By the definition of $\bar{\mu}$, can be mapped $k$-homomorphically onto a non-zero semiring of $\mu$, and therefore $R$ is in $\bar{\mu}$, and thus $\bar{\mu}$ satisfies $(\mathrm{F})$.

Example 4. Let $\mu$ be the class of all strongly austere semirings over the class $\Im$. It is clear that the class $\mu$ satisfies (E). If the class $\bar{\mu}$ is defined as the set of all semirings $R \in \Im$ such that every non-zero subtractive ideal of $R$ can be mapped $k$-homomorphically onto some non-zero semiring of $\mu$, then the class $\bar{\mu}$ satisfies (E) and (F). By theorem 7 we can then obtain a semiradical class $\rho_{\bar{\mu}}$.

Thus, if we start with a class $\mu$ of semirings over the class $\Im$ that satisfies (E), we can build it up to a class $\bar{\mu}$ which satisfies both (E) and (F), and by theorem 7 we can then obtain a semiradical class $\rho_{\bar{\mu}}$. We call this class the upper semiradical determined by $\mu$. The use of the word upper is justified by the following:

Proposition 6. If $\mu$ is a class of semiring over $\Im$ which satisfies (E) and if $\rho$ is a semiradical class over $\Im$ for which all the semirings in $\mu$ are $\rho$-semisimple, then $\rho$ is a subset of the upper semiradical determined by $\mu$.

Proof. By Theorem 7, the class of all $\rho$-semisimple semirings satisfies (F), so this class contains all semirings of $\bar{\mu}$, and thus $\rho \subset \rho_{\bar{\mu}}$.

In the following example 5 we will show that the Jacobson semiradical over a certain restricted class $\chi$ is the upper semiradical determined by the class of all regular strongly austere semiring over $\chi$. The reader is referred to the appendix for necessary background results. 
Example 5. Let $\chi$ be the class of all semiring $R$ with identity 1 over the class $\Im$, for which every subtractive ideal $I$ of $R$ satisfies the following conditions:

(1) $I(r / K)$ is subtractive for every regular maximal subtractive left ideal $K$ of $R$

(2) If $r e / L_{I}(i / K)=r^{\prime} e / L_{I}(i / K)$ then $r e / L_{R}(i / K)=r^{\prime} e / L_{R}(i / K)$ where $K$ is a regular maximal subtractive left ideal of $I, e i / K=i / K$ and $e, i \in I$.

By theorem 8, the class of Jacobson semiradical over $\chi, J S=\{J S(R): R \in \chi\}$ forms a semiradical class. Moreover, proposition 7 implies that $J S(R)=\cap_{\alpha \in A} K_{\alpha}$, where $\left\{K_{\alpha}: \alpha \in A\right\}$ is the set of all regular maximal subtractive left ideal. Let $\lambda$ be the class of all regular strongly austere semirings over $\chi$. It is clear that the class $\lambda$ satisfies $(\mathrm{E})$, we can build it up to a class $\bar{\lambda}$ which satisfies both (E) and (F), and by theorem 7 we can then obtain a semiradical class $\rho_{\bar{\lambda}}$. If $R \in \lambda$, then $J S(R)=0$. By proposition 6 $J S \subset \rho_{\bar{\lambda}}$. Let $R \in \rho_{\bar{\lambda}}$. If $R \notin J S$, then there exists a regular maximal subtractive ideal $K_{\alpha}$. Hence $R / K_{\alpha} \in \bar{\lambda}$. Therefore $R / J S(R) \notin \rho_{\bar{\lambda}}$. Thus $R \notin \rho_{\bar{\lambda}}$. This contradiction proves that $R \in J S$. Therefore $J S=\rho_{\bar{\lambda}}$.

\section{Appendix}

Here, we recall some necessary definition and results which we have used in the main text.

Definition 5. For a semiring $R$ the Jacobson semiradical $J S(R)$ is the annihilator of $R$ in $\tau$; i.e., $J S(R)=L_{R}(\tau)$ where $\tau$ is the class of all regular strongly austere left $R$-semimodules.

Proposition 7. For any semiring $R$ the following properties hold:

(1) $J S(R)$ is the intersection of all of the rejects of regular strongly austere left $R$ semimodules.

(2) $J S(R)$ is the intersection of all of the strong rejects of regular strongly austere left $R$-semimoudles.

(3) $J S(R)$ is the intersection of all of the regular modular maximal subtractive left ideals of $R$.

Theorem 8. The class $J S=\{J S(R): R \in v\}$ froms a semiradical class, where $v$ is the class of semirings $R$ for which every subtractive ideal $I$ of $R$ satisfies the following conditons:

(1) If $i \underset{L_{R}(r / K)}{\equiv} i^{\prime}$ then $i \underset{L_{R}(r / K) \cap I}{\equiv} i^{\prime}$ where $K$ is a regular modular maximal subtractive left ideal of $R, i, i^{\prime} \in I$ and $r \in R$.

(2) $I(r / K)$ is subtractive for every regular modular maximal subtractive left ideal $K$ of $R$.

(3) If re $/ L_{I}(i / H)=r^{\prime} e / L_{I}(i / H)$ then $r e / L_{R}(i / H)=r^{\prime} e / L_{R}(i / H)$ where $H$ is a regular modular maximal subtractive left ideal of $I$, ei $/ H=i / H$, and $e, i \in I$. 
(For the proofs of the above results, please contact the author directly. They are included in a paper submitted for publication)

\section{References}

[1] Huda M. J. Al-Thani, Congenerators of semimodules and the notion of rejects II: Rejects of semimoules. AMSE Journal, to appear.

[2] N. J. Divinsky, Rings and Radicals. George Allen \& Unwin, London, 1965.

[3] J. S. Golan, The Theory of Semirings with Applications in Mathematics and Theoretical Computer Science, Pitman monographs and surveys in pure and applied mathematics. Longman Scientific \& Technical, Harlow UK, 1992.

[4] Michihiro Takahashi, On the bordism categories III, Functors Hom and $\otimes$ for semimodules, Math. Sem. Notes 10(1982), 2M-236

Mathmeatics Department, Faculty of Science, University of Qatar, Doha, Qatar.

E-mail: h.m.althani@qu.edu.qa 